PROCEEDINGS OF THE

AMERICAN MATHEMATICAL SOCIETY

Volume 137, Number 1, January 2009, Pages 393-400

S 0002-9939(08)09507-5

Article electronically published on September 3, 2008

\title{
ON THE FARRELL COHOMOLOGY OF THE MAPPING CLASS GROUP OF NON-ORIENTABLE SURFACES
}

\author{
GRAHAM HOPE AND ULRIKE TILLMANN
}

(Communicated by Paul Goerss)

\begin{abstract}
We study the unstable cohomology of the mapping class groups $\mathcal{N}_{g}$ of non-orientable surfaces of genus $g$. In particular, we determine for all genus $g$ and all primes $p$ when the group $\mathcal{N}_{g}$ is $p$-periodic.

To this purpose we show that $\mathcal{N}_{g}$ is a subgroup of the mapping class group $\Gamma_{g-1}$ of an orientable surface of genus $g-1$ and deduce that $\mathcal{N}_{g}$ has finite virtual cohomological dimension. Furthermore, we describe precisely which finite groups of odd order are subgroups of $\mathcal{N}_{g}$.
\end{abstract}

\section{INTRODUCTION}

Because of their close relation to moduli spaces of Riemann surfaces, the mapping class groups of orientable surfaces have been the focus of much mathematical research for a long time. Less well studied is the mapping class group of nonorientable surfaces, although recently the study of mapping class groups has also been extended to the non-orientable case. This paper contributes to this programme. While Wahl [W] proved the analogue of Harer's (co)homology stability in the non-oriented case, we concentrate here on the unstable part of the cohomology. In particular, we study the question of $p$-periodicity.

Recall that a group $G$ of finite virtual cohomological dimension $(v c d)$ is said to be $p$-periodic if the $p$-primary component of its Farrell cohomology ring, $\hat{H}^{*}(G, \mathbf{Z})_{(p)}$, contains an invertible element of positive degree. Farrell cohomology extends Tate cohomology of finite groups to groups of finite $v c d$. In degrees above the $v c d$ it agrees with the ordinary cohomology of the group. For the mapping class group in the oriented case, the question of $p$-periodicity has been examined by Xia $[\underline{\mathrm{X}}]$ and by Glover, Mislin and Xia GMX. Here we determine exactly for which genus and prime $p$ the non-orientable mapping class groups are $p$-periodic. In the process we also establish that these groups are of finite cohomological dimension and present a classification theorem for finite group actions on non-orientable surfaces.

Let $N_{g}$ be a non-orientable surface of genus $g$, i.e. the connected sum of $g$ projective planes. The associated mapping class group $\mathcal{N}_{g}$ is defined to be the group of connected components of the group of homeomorphisms of $N_{g}$. The mapping class groups of the projective plane and the Klein bottle are well known to be the trivial group and the Klein 4-group respectively, namely

$$
\mathcal{N}_{1}=\{e\} \quad \text { and } \quad \mathcal{N}_{2}=C_{2} \times C_{2} .
$$

Received by the editors September 25, 2007, and, in revised form, January 18, 2008.

2000 Mathematics Subject Classification. Primary 57M60; Secondary 20J05, 57S05. 
Throughout this paper we may therefore assume that $g \geq 3$. Our main result can now be stated as follows.

Theorem 1.1. $\mathcal{N}_{g}$ is not p-periodic in the following two cases.

(1) Assume $p=2$. Then $\mathcal{N}_{g}$ is not $p$-periodic.

(2) Assume $p$ is odd and $g \equiv 2(\bmod p)$. Write $g=l p+2$ with $l=k p-t$ for some $k>0$ and $0 \leq t<p$. If $k>t-2$, then $\mathcal{N}_{g}$ is not p-periodic.

In all other cases $\mathcal{N}_{g}$ is p-periodic.

In particular, $\mathcal{N}_{g}$ is $p$-periodic whenever $p$ is odd and $g$ is not equal to $2 \bmod p$. On the other hand, for odd $p, \mathcal{N}_{g}$ is not $p$-periodic for all $g>p^{3}$ with $g$ equal to 2 $\bmod p$.

In outline, we will first show that the mapping class group $\mathcal{N}_{g}$ of a non-orientable surface of genus $g$ is a subgroup of the mapping class group $\Gamma_{g-1}$ of an orientable surface of genus $g-1$. Many properties of $\Gamma_{g-1}$ are thus inherited by $\mathcal{N}_{g}$. In particular it follows that $\mathcal{N}_{g}$ is of finite virtual cohomological dimension and its Farrell cohomology is well-defined. We then recall that a group $G$ is not $p$-periodic precisely when $G$ has a subgroup isomorphic to $C_{p} \times C_{p}$, the product of two cyclic groups of order $p$. Motivated by this we prove a classification theorem for actions of finite groups on non-orientable surfaces. From this it is straightforward to deduce necessary and sufficient conditions for $C_{p} \times C_{p}$ to act on $N_{g}$. Finally, we discuss some open questions.

\section{Preliminaries}

Let $\Sigma_{g-1}$ be a closed orientable surface of genus $g-1$, embedded in $\mathbb{R}^{3}$ such that $\Sigma_{g-1}$ is invariant under reflections in the $x y$-, $y z$-, and $x z$-planes. Define a (orientation-reversing) homeomorphism $J: \Sigma_{g-1} \rightarrow \Sigma_{g-1}$ by

$$
J(x, y, z)=(-x,-y,-z) .
$$

$J$ is reflection in the origin. Under the action of $J$ on $\Sigma_{g-1}$, the orbit space is homeomorphic to a non-orientable surface $N_{g}$ of genus $g$ with associated orientation double cover

$$
p: \Sigma_{g-1} \longrightarrow N_{g} \text {. }
$$

Let $\Gamma_{g-1}^{ \pm}$denote the extended mapping class group, i.e. the group of connected components of the homeomorphisms of $\Sigma_{g-1}$, not necessarily orientation-preserving. $\Gamma_{g-1}$ as usual will denote its index 2 subgroup corresponding to the orientation preserving homeomorphisms.

Birman and Chillingworth $\mathrm{BC}$, give the following description of the mapping class group $\mathcal{N}_{g}$. Let $C\langle J\rangle \subset \Gamma_{g-1}^{ \pm}$be the group of connected components of

$$
S(J):=\left\{\varphi \in \operatorname{Homeo}\left(\Sigma_{g-1}\right) \mid \exists \tilde{\varphi} \text { isotopic to } \varphi \text { such that } \tilde{\varphi} J=J \tilde{\varphi}\right\},
$$

the subgroup of homeomorphisms that commute with $J$ up to isotopy. By definition, $J$ generates a normal subgroup of $C\langle J\rangle$. Birman and Chillingworth identify the quotient group with the mapping class group of the orbit space $N_{g}=\Sigma_{g-1} /\langle J\rangle$,

$$
\mathcal{N}_{g} \cong \frac{C\langle J\rangle}{\langle J\rangle}
$$

The following result has proved very useful, as many properties of $\Gamma_{g-1}$ are inherited by $\mathcal{N}_{g}$. 
Key-Lemma 2.1. $\mathcal{N}_{g}$ is isomorphic to a subgroup of $\Gamma_{g-1}$.

Proof. Consider the projection

$$
\pi: C\langle J\rangle \longrightarrow \frac{C\langle J\rangle}{\langle J\rangle} \cong \mathcal{N}_{g}
$$

For a subgroup $G$ of $\mathcal{N}_{g}$ write

$$
\pi^{-1}(G)=G^{+} \cup G^{-} \subset C\langle J\rangle,
$$

where

$$
G^{+}:=\pi^{-1}(G) \cap \Gamma_{g-1} \text { and } G^{-}:=\pi^{-1}(G) \cap\left(\Gamma_{g-1}^{ \pm} \backslash \Gamma_{g-1}\right) .
$$

Note that $G^{-}=J G^{+}$. We claim that $\left.\pi\right|_{G^{+}}: G^{+} \rightarrow G$ is an isomorphism. Indeed, injectivity holds, as the only non-zero element $J$ in the kernel of $\pi$ is not an element of $G^{+}$. Surjectivity is also immediate as every element in $G$ has exactly two preimages under $\pi$ which differ by $J$. Thus exactly one of them is an element in the orientable mapping class group $\Gamma_{g-1}$, that is, an element of $G^{+}$.

Recall that Farrell cohomology is defined only for groups of finite virtual cohomological dimension.

Corollary 2.2. The non-orientable mapping class group $\mathcal{N}_{g}$ has finite virtual cohomological dimension with

$$
v c d \mathcal{N}_{g} \leq 4 g-9 .
$$

Proof. The mapping class group $\Gamma_{g-1}$ is virtually torsion free. Furthermore, from Harer $\left[\mathrm{H}\right.$ ], we know that $\Gamma_{g-1}$ is of finite virtual cohomological dimension $4(g-1)-5$. Hence every subgroup of $\Gamma_{g-1}$ will also have finite virtual cohomological dimension with $v c d$ less than or equal to $4 g-9$, cf. [Br, Exercise 1, p. 229]. The corollary now follows from the Key-Lemma.

\section{Classifying finite group actions on $N_{g}$}

The purpose of this section is to give necessary and sufficient criteria for when a finite group is isomorphic to a subgroup of $\mathcal{N}_{g}$. For the purpose of this paper we are only interested in groups of odd order.

Theorem 3.1. Let $N_{g}$ denote a non-orientable surface of genus $g$, and let $A$ be a finite group of odd order. Then $A$ is isomorphic to a subgroup of $\operatorname{Homeo}\left(N_{g}\right)$ if and only if $A$ has partial presentation

$$
\left\langle c_{1}, \ldots, c_{h}, y_{1}, \ldots, y_{t} \mid \ldots\right\rangle
$$

such that

(1) $h \geq 1$;

(2) $\prod_{j=1}^{h} c_{j}^{2} \prod_{i=1}^{t} y_{i}=1$;

(3) the order of $y_{i}$ in $A$ is $m_{i}$;

(4) the Riemann-Hurwitz equation holds:

$$
g-2=|A|(h-2)+|A| \sum_{i=1}^{t}\left(1-\frac{1}{m_{i}}\right) .
$$

The proof of the theorem is an application of the theory of covering spaces. Different versions of the theorem can be found in the literature; see for example T]. For completeness and convenience for the reader we include a proof. 
Proof. Assume $A$ has a partial presentation of the form described in the theorem, and let $N_{h}$ be a non-orientable surface of genus $h \geq 1$. Represent $N_{h}$ as a $2 h$-sided polygon with sides to be identified in pairs, where the polygon is bounded by the cycle $c_{1} c_{1} c_{2} c_{2} \ldots c_{h} c_{h}$. At a vertex add $t$ (non-intersecting) loops $y_{1}, \ldots, y_{t}$ so that the resulting 2 -cells bounded by $y_{1}, \ldots, y_{t}$ are mutually disjoint and are contained in the polygon; see Figure 1. Choose a direction for each of the loops $y_{1}, \ldots, y_{t}$ and call the resulting one-vertex graph $G$. Note that we can give $N_{h}$ the structure of a CW-complex so that $G$ is cellularly embedded in $N_{h}$. A covering graph $\tilde{G}$ is

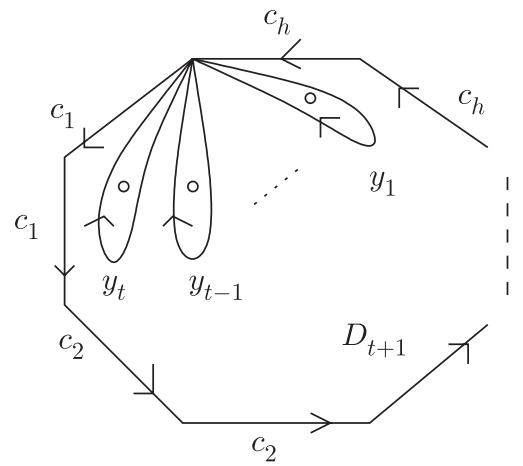

Figure 1. A non-orientable surface of genus $h$.

obtained from $G$ as follows. Its vertex set and edge set are $A$ and $E \times A$ respectively, where $E$ is the edge set of the graph $G$. If $e$ is an edge of $G$, then the edge $(e, a)$ of $\tilde{G}$ runs from the vertex $a$ to the vertex ae. The forgetful map of graphs $p: \tilde{G} \rightarrow G$ is a covering map which we now extend to a branched covering map $p: S \rightarrow N_{h}$ of surfaces as follows.

Label the regions of $N_{h}$ as $D_{1}, D_{2}, \ldots, D_{t}$ and $D_{t+1}$, where $D_{1}, D_{2}, \ldots, D_{t}$ are bounded by the loops $y_{1}, y_{2}, \ldots, y_{t}$ and $D_{t+1}$ is the remaining region. For each cycle $C$ in $G, p^{-1}(C)$ is a collection of cycles in $\tilde{G}$. The cycles $y_{i}$ have $\frac{|A|}{m_{i}}$ corresponding cycles in $\tilde{G}$, for each $i \in\{1, \ldots, t\}$. Finally, the cycle $c_{1} c_{1} \ldots c_{h} c_{h} y_{1} \ldots y_{t}$, bounding $D_{t+1}$, has $|A|$ cycles above it in $\tilde{G}$, because the order of $\prod_{j=1}^{h} c_{j}^{2} \prod_{i=1}^{t} y_{i}$ in $A$ is 1 .

To each of these cycles in $\tilde{G}$ attach a 2-cell. Then extend $p$ by mapping the interior of each 2-cell onto the interior of the 2-cell $D_{n}$ by using the maps $z \rightarrow z^{d}$, where $d=m_{i}$ for $n \in\{1, \ldots, t\}$ and $d=1$ for $n=t+1$. We obtain a surface $S$ which admits a CW-structure with $\tilde{G}$ cellularly embedded in $S$.

We now argue by contradiction that $S$ is non-orientable. Suppose that $S$ is an orientable surface, and let $A^{0} \subset A$ be the subgroup of homeomorphisms which preserve the orientation. Now $A^{0} \neq A$ since $N_{g}$ is non-orientable. So, $A^{0}$ is a subgroup of index 2 in $A$, which contradicts our assumption that $A$ is of odd order. So $S$ is non-orientable. Finally, its genus $g$ is determined by the Riemann-Hurwitz formula, condition (4).

Conversely, assume $A$ acts on the non-orientable surface $S=N_{g}$. As $A$ is of odd order, $A$ acts without reflections and its singular set is discrete. Thus the quotient map $p: S \rightarrow S / A$ is a branched covering, and $S / A$ is a non-orientable surface of genus $h \geq 1$. Represent $S / A$ as a $2 h$-sided polygon with sides $c_{1}, c_{1}, c_{2}, c_{2}, \ldots, c_{h}, c_{h}$ to be identified in pairs, and in which the branch points of $p$ are in the interior of 
the polygon. Now add mutually disjoint loops $y_{1}, \ldots, y_{t}$ around each branch point, all starting at the same vertex as indicated in Figure 1. Let us call the resulting one-vertex graph $G$. Its inverse image $p^{-1}(G)$ is a Cayley graph for the group $A$ : vertices correspond to the elements of $A$ and at each vertex there are $2(h+t)$ directions corresponding to generators $c_{i}$ and $y_{i}$. The three conditions for the partial presentations are easily verified. First note that $h$ is positive as $S / A$ is non-orientable. As $\prod_{j=1}^{h} c_{j}^{2} \prod_{i=1}^{t} y_{i}=1$ is a closed curve in $S / A$, so it is in $S$ and hence must represent the identity in $A$. The order $m_{i}$ of $y_{i}$ is precisely the branch number of the singular point that $y_{i}$ encircles. Thus the formula in condition (4) follows from the Riemann-Hurwitz equation.

As we are interested in subgroups of the mapping class group, we state the following result, which is well-known at least for orientable surfaces.

Theorem 3.2. A finite group $G$ is a subgroup of the mapping class group $\mathcal{N}_{g}$ if and only if it is a subgroup of $\operatorname{Homeo}\left(N_{g}\right)$.

Proof. If $G$ is a finite subgroup of $\mathcal{N}_{g}$, then it follows by the Nielsen realisation problem for non-orientable surfaces $[\mathrm{K}]$ that $G$ lifts to a subgroup of Homeo $\left(N_{g}\right)$. Conversely, let $G$ be a finite subgroup of $\operatorname{Homeo}\left(N_{g}\right)$. An application of the Lefschetz Fixed Point Formula shows that for all $g \geq 3$, any element of finite order in $\operatorname{Homeo}\left(N_{g}\right)$ cannot be homotopic to the identity. Hence the kernel of the canonical projection $\operatorname{Homeo}\left(N_{g}\right) \rightarrow \mathcal{N}_{g}$ when restricted to a finite subgroup $G \in \operatorname{Homeo}\left(N_{g}\right)$ must be trivial.

Theorem 3.1 and Theorem 3.2 together imply that a finite group $A$ of odd order is a subgroup of the mapping class group $\mathcal{N}_{g}$ if and only if it has partial presentation such that conditions (1) to (4) in Theorem 3.1 hold.

\section{The $p$-Periodicity OF $\mathcal{N}_{g}$}

Using the result of the previous section we can now prove our main result. Theorem 1.1 is equivalent to the following three lemmata. Recall (see e.g. $\overline{\mathrm{Br}}$, Theorem 6.7]) that a group of finite $v c d$ is $p$-periodic if and only if it does not contain an elementary abelian subgroup of rank two.

Lemma 4.1. $\mathcal{N}_{g}$ is not 2-periodic.

Proof. It will suffice to exhibit a subgroup of $\mathcal{N}_{g}$ isomorphic to $C_{2} \times C_{2}$. Let $R_{1}$ and $R_{2}$ be homeomorphisms of $\Sigma_{g-1}$ (embedded in $\mathbb{R}^{3}$ as before) which are rotations by $\pi$, given by the formulae

$$
\begin{aligned}
& R_{1}(x, y, z)=(-x,-y, z), \\
& R_{2}(x, y, z)=(x,-y,-z) .
\end{aligned}
$$

Clearly, $J, R_{1}$ and $R_{2}$ are all involutions. For $g \geq 3$ the induced actions on the first homology groups $H_{1}\left(\Sigma_{g-1}\right)$ are non-trival and all different; they define non-trivial, distinct elements of order two in $\Gamma_{g-1}^{ \pm}$. From their defining formulas it is clear that they commute with each other. Hence, they generate a subgroup

$$
H=C_{2} \times C_{2} \times C_{2} \subset C\langle J\rangle \subset \Gamma_{g-1}^{ \pm},
$$

and thus

$$
\pi(H) \cong C_{2} \times C_{2} \subset \frac{C\langle J\rangle}{\langle J\rangle} \cong \mathcal{N}_{g}
$$


Thus $\mathcal{N}_{g}$ is never 2-periodic,

Lemma 4.2. Let $p$ be odd. $\mathcal{N}_{g}$ is not p-periodic in the following three cases.

(1) If $g=l p+2$ and $l=k p$ where $k>0$, then $\mathcal{N}_{g}$ is not $p$-periodic.

(2) If $g=l p+2$ and $l=k p-1$ where $k>0$, then $\mathcal{N}_{g}$ is not p-periodic.

(3) If $g=l p+2, l=k p-t$ and $k>t-2$ where $2 \leq t<p$, then $\mathcal{N}_{g}$ is not p-periodic.

Proof. In all three cases we will use Theorem 3.1 (and Theorem 3.2) to exhibit subgroups $C_{p} \times C_{p} \subset \mathcal{N}_{g}$. Hence, in these cases $\mathcal{N}_{g}$ is not $p$-periodic.

Case (1): Let $h:=k+2 \geq 3$. A presentation of $A=C_{p} \times C_{p}=\left\langle c_{1}\right\rangle \times\left\langle c_{2}\right\rangle$ can now be given as follows:

$$
A=\left\langle c_{1}, \ldots, c_{h} \mid c_{3}=c_{1}^{p-1} c_{2}^{p-1}, c_{4}=\ldots=c_{h}=1, c_{1} c_{2}=c_{2} c_{1}, c_{1}^{p}=c_{2}^{p}=1\right\rangle .
$$

One checks that the four conditions of Theorem 3.1 are satisfied; here

$$
g-2=p^{2}(h-2) .
$$

Case (2): Let $h:=k+1 \geq 2$. A presentation of $A=C_{p} \times C_{p}=\left\langle c_{1}\right\rangle \times\left\langle c_{2}\right\rangle$ is given by

$$
A=\left\langle c_{1}, \ldots, c_{h}, y_{1} \mid y_{1}=c_{1}^{p-2} c_{2}^{p-2}, c_{3}=\ldots=c_{h}=1, c_{1} c_{2}=c_{2} c_{1}, c_{1}^{p}=c_{2}^{p}=1\right\rangle .
$$

Again one easily checks that the four conditions of Theorem 3.1 are satisfied; in this case

$$
g-2=p^{2}(h-2)+p^{2}\left(1-\frac{1}{p}\right) .
$$

Case (3): Let $h:=k+2-t \geq 1$. As also $t \geq 2$, a presentation of $A=C_{p} \times C_{p}=$ $\left\langle y_{1}\right\rangle \times\left\langle y_{2}\right\rangle$ is now given by

$$
\begin{gathered}
A=\left\langle c_{1}, \ldots, c_{h}, y_{1}, y_{2}, \ldots, y_{t}\right| c_{1}=y_{1}^{\frac{p-1}{2}} y_{2}^{\frac{p-1}{2}} \ldots y_{t}^{\frac{p-1}{2}} \\
\left.y_{2}=y_{3}=\ldots=y_{t}, c_{2}=c_{3}=\ldots=c_{h}=1, y_{1} y_{2}=y_{2} y_{1}, y_{1}^{p}=y_{2}^{p}=1\right\rangle .
\end{gathered}
$$

This presentation satisfies the conditions of Theorem 3.1 with

$$
g-2=p^{2}(h-2)+p^{2} t\left(1-\frac{1}{p}\right) .
$$

Hence in all these three cases, i.e. whenever condition (2) of Theorem 1.1 holds, the mapping class group $\mathcal{N}_{g}$ is not $p$-periodic.

Lemma 4.3. Let $p$ be odd and assume that $g$ does not satisfy any of the three conditions of Lemma 4.2; then $\mathcal{N}_{g}$ is p-periodic.

Proof. Let $p$ be odd and suppose that there exists a subgroup $A=C_{p} \times C_{p}$ contained in $\mathcal{N}_{g}$. Then by Theorem 3.1 (and Theorem 3.2), $A$ acts on $N_{g}$ and the RiemannHurwitz Formula must be satisfied for some $h \geq 1$ where $h$ is the genus of the quotient surface $N_{g} / A$. ( $h$ cannot be zero as the sphere cannot arise as the quotient of a non-oriented surface.) Let $s$ be the number of singular points of the action of $A$ on $N_{g}$, and let $a$ be an element in the stabiliser of some singular point $x$. By Key-Lemma 2.1, $a$ lifts to an element of $\Gamma_{g-1}$ and by the Nielsen realization problem to a homeomorphism, also denoted by $a$, of $\Sigma_{g-1}$. The singular point $x$ lifts to two points in $\Sigma_{g-1}$, and under the action of $a$ these form two separate orbits as the group $A$ and hence the element $a$ are of odd order. So $a$ is in the stabiliser of these two points and therefore must act freely on the tangent planes at these points 
(for otherwise $a$ would be homotopic to a homeomorphism that fixes a whole disk, but all such homeomorphisms are well-known to give rise to elements of infinite order in the mapping class group). This also implies that the action of $a$ on the tangent plane at $x$ in $N_{g}$ is free. It follows that the stabiliser of each singular point is isomorphic to $C_{p}$ as these are the only non-trivial subgroups of $A$ that are also subgroups of $\mathrm{GL}_{2}(\mathbb{R})$. So by the Riemann-Hurwitz equation, for some $h \geq 1$,

$$
g-2=p^{2}(h-2)+p s(p-1) .
$$

From this it follows that $g=l p+2$ for some $l \geq 1$, and furthermore that $l=$ $p(h-2+s)-s$. Note that $l=-s(\bmod p)$. Now write $s=q p+t$ for some $q \geq 0$ and $0 \leq t<p$. Then $l=p(h+q(p-2)-2+t)-t$. Thus we are in the situation of Lemma 4.2. Indeed, as $h \geq 1$, we can write $l=k p-t$ with $k=h+q(p-2)+t-2>t-2$. Lemma 4.3 follows from this.

Remark 4.4. A group is $p$-periodic if and only if it does not contain a subgroup isomorphic to $C_{p} \times C_{p}$. Therefore, any subgroup of a $p$-periodic group is $p$-periodic. Hence by the Key-Lemma 2.1, the $p$-periodicity of any $\Gamma_{g-1}$ implies the $p$-periodicity of $\mathcal{N}_{g}$. (In particular, as $\Gamma_{g-1}$ is always $p$ periodic for odd $p$ and $g$ not equal to 2 $\bmod p$, so is $\mathcal{N}_{g}$.) However, comparing our results with those of Xia [X], we note here that the converse is false. For example, when $p=5$ and $g=7, \Gamma_{6}$ is not $p$-periodic but $\mathcal{N}_{7}$ is. However, for a fixed $p$ there are at most finitely many such $g$ where $\Gamma_{g-1}$ is not $p$-periodic but $\mathcal{N}_{g}$ is.

\section{THE $p$-PERIOD AND OTHER OPEN QUESTIONS}

We will briefly discuss three questions that arise from our study.

5.1. The $p$-period. Recall that the $p$-period $d$ of a $p$-periodic group $G$ is the least positive degree of an invertible element in its Farrell cohomology group $\hat{H}^{*}(G, \mathbf{Z})_{(p)}$. The question thus arises as to what the $p$-period of $\mathcal{N}_{g}$ is when $\mathcal{N}_{g}$ is $p$-periodic.

For any group $G$ of finite $v c d$, an invertible element in $\hat{H}^{*}(G, \mathbf{Z})_{(p)}$ restricts to an invertible element in the Farrell cohomology of any subgroup of $G$. Thus the $p$-period of a subgroup divides the $p$-period of $G$.

The main result of [GMX] is that for all $g$ such that $\Gamma_{g-1}$ is $p$-periodic, the $p$ period divides $2(p-1)$. Hence for all such $g$, the $p$-period of $\mathcal{N}_{g}$ also divides $2(p-1)$. However, as we noted above, there are pairs $p$ and $g$ for which $\mathcal{N}_{g}$ is $p$-periodic but $\Gamma_{g-1}$ is not. We expect that the methods of [GMX] can be pushed to cover also these cases. It remains also to find lower bounds for the $p$-period.

5.2. Punctured mapping class groups. In the oriented case Lu [L1], [L2] has studied the $p$-periodicity of the mapping class groups with marked points, and proved that they are all $p$-periodic of period 2 . One might expect a similar result to hold for the mapping class group of non-orientable surfaces with marked points.

5.3. The virtual cohomological dimension. We have established in Corollary 2.2 that $\mathcal{N}_{g}$ has finite virtual cohomological dimension and that this dimension is less than or equal to $4 g-9$. It seems an interesting project to determine the $v c d$ of $\mathcal{N}_{g}$. 


\section{REFERENCES}

[BC] Birman, J.S.; Chillingworth, D.R.J.: On the homeotopy group of a non-orientable surface, Proc. Camb. Phil. Soc. 71, 437-448 (1972). MR0300288 (45:9334)

[Br] Brown, K.S.: Cohomology of groups, Graduate Texts in Mathematics 87, Springer-Verlag, New York (1982). MR672956 (83k:20002)

[GMX] Glover, H.H.; Mislin, G.; Xia, Y.: On the Farrell cohomology of mapping class groups, Invent. Math. 109, 535-545 (1992). MR1176203 (93k:57034)

[H] Harer, J.: The virtual cohomological dimension of the mapping class group of an orientable surface, Invent. Math. 84, 157-176 (1986). MR830043 (87c:32030)

[K] Kerckhoff, S.: The Nielson realization problem, Ann. of Math. (2) 117, 235-263 (1983). MR690845 (85e:32029)

[L1] Lu, Q.: Periodicity of the punctured mapping class group, J. Pure Appl. Algebra 155, 211-235 (2001). MR1801416 (2002b:57018)

[L2] Lu, Q.: Farrell cohomology of low genus pure mapping class groups with punctures, Alg. Geom. Top. 2, 537-562 (2002). MR1917066 (2003k:55005)

[T] Tucker, T.: Finite groups acting on surfaces and the genus of a group, J. Combin. Theory Ser. B 34, 82-98 (1983). MR701174 (85b:20055)

[W] Wahl, N.: Homological stability for the mapping class groups of non-orientable surfaces, Invent. Math. 171, 389-424 (2008). MR 2367024

[X] Xia, Y.: The p-periodicity of the mapping class group and the estimate of its p-period, Proc. Amer. Math. Soc. 116, 1161-1169 (1992). MR1104406 (93b:57032)

Mathematical Institute, Oxford University, Oxford OX1 3LB, United Kingdom

E-mail address: hope@maths.ox.ac.uk

Mathematical Institute, Oxford University, Oxford OX1 3LB, United Kingdom

E-mail address: tillmann@maths.ox.ac.uk 